\title{
Forrajeo de Didelphis pernigra (Mammalia: Didelphidae) en un área suburbana de la Sabana de Bogotá, Colombia
}

Viviana Barrera-Niño ${ }^{1 *}$ y Francisco Sánchez ${ }^{1}$

Introduction: In Colombia, there is widespread transformation of natural ecosystems, especially in the Andean region. However, the impacts of those transformations have been poorly studied. Optimal patch theory was used to evaluate the foraging behavior and movement patterns in the white-eared opossum (Didelphis pernigra) in the Sabana de Bogotá, a plateau at about 2,600 $\mathrm{m}$ in the Colombian Andes. The study site was dominated by an exotic grass (Cenchrus clandestinum), and little is known about possible impacts of this exotic species on native fauna.Thus, we analyzed whether this grass the foraging behavior and movements of $D$. pernigra. To test this, we used the giving up density (GUD) technique, which uses the amount of food left in a feeding patch to assess the forager's habitat perception. We also tested whether this exotic grassland acted as a barrier to movements to and from an area of native and exotic trees. Finally, we measured the influence of moon illumination, air temperature, and precipitation on the oppossum's foraging behavior.

Methodology: We established two approximately parallel transects in a suburban area (Fig. 1). Foraging stations were located 30 to 40 meters apart on each transect. Each station consisted of a feeder made of a cylindrical plastic container with $150 \mathrm{ml}$ of sugar water in a 1:3 proportion. Each container also contained 60 glass spheres to generate diminishing returns on the harvest rate. The oppossums were habituated to the feeders for 15 days, and then GUDs were measured for 30 days. Feeders were filled before sunset and checked the following morning.

Results: Oppossums foraged at least $240 \mathrm{~m}$ into the grasslands from the trees along the river. Air temperature and precipitation did not influence their foraging behavior (Fig. 2). There was, however, temporal variation in foraging activity, and at least some of this was due to a negative effect of lunar illumination (Fig. 3).

Discussion and Conclusions: Our results indicate that the white-eared opossum is a tolerant species able to exploit human-created habitats. This finding is in agreement with reports on other species of Didelphis that they are tolerant of moderate human interventions. We surmise that this species' ability to exploit the open ecosystems of the paramo has made it possible for it to effectively use the grasslands on the study site. The only abiotic factor that was found to be influential was moon illumination. Darker nights gave lower GUD's indicating that the foragers perceived decreased predation risk. Finally, we argue that efforts should be made to prevent the local extinction of this opossum in disturbed areas of Bogotá since it could aid in the conservation of ecological functions in those areas.

Key words: Andes, giving-up density (GUD), habitat selection, optimal patch use, urban ecology.

\section{Resumen}

Aplicamos la teoría de uso óptimo de parches para evaluar los comportamientos de forrajeo e inferir las posibilidades de movimiento del tlacuache de orejas blancas o fara,

${ }^{1}$ Grupo Integrado de Investigaciones en Química y Biología, Programa de Biología Aplicada, Facultad de Ciencias Básicas y Aplicadas, Universidad Militar Nueva Granada. Cajicá, km 2 vía Cajicá-Zipaquirá, Colombia. E-mail: vivibani@gmail. com (VBN), fasbos@gmail.com (FS)

*Corresponding author 
Didelphis pernigra en un área dominada por un pasto exótico (Cenchrus clandestinum), en la Sabana de Bogotá, Andes colombianos. Evaluamos si el pastizal actuaba como una barrera para los movimientos del fara, y también el posible efecto de la distancia a un área con árboles nativos y exóticos cerca al río Bogotá, la iluminación de la luna, la

temperatura del aire y la precipitación. Los faras se adentraron al menos $240 \mathrm{~m}$ en el pastizal, y la distancia respecto a la zona arbolada, la temperatura del aire y la precipitación no afectaron su forrajeo. La iluminación de la luna se relacionó negativamente con el forrajeo de los faras, probablemente al incrementar el costo percibido de depredación. Nuestros resultados indican que el fara es una especie tolerante capaz de aprovechar espacios creados por el ser humano, y argumentamos que deberían hacerse esfuerzos para evitar que este marsupial desaparezca de las áreas altamente disturbadas de la Sabana de Bogotá, dado que podría ayudar en la conservación de funciones ecológicas en esas áreas.

Palabras clave: Andes, densidad de abandono (DDA), ecología urbana, uso óptimo de parches, selección de hábitat.

Una de las principales amenazas para la biodiversidad es la transformación de los ecosistemas naturales, que puede causar la destrucción y fragmentación de ecosistemas y reducir la conectividad para las poblaciones remanentes (Pullin 2002). En Colombia es evidente la transformación de los ecosistemas naturales y es particularmente notable en la región andina (Etter y van Wyngaarden 2000). Esto se debe principalmente a que la mayor parte de la población humana (66\% de la población, 30 millones de personas) en Colombia se ubica en los Andes (Etter y Villa 2000). Por otra parte, los Andes han sido reconocidos como la región natural con la mayor riqueza de especies de flora y fauna del país y también presenta un alto nivel de endemismos (Armenteras et al. 2003). Lo anterior sugiere que deben hacerse esfuerzos especiales para proteger este patrimonio natural, pero dada la alta cantidad de humanos y nivel de disturbio presente en los Andes colombianos, dichos esfuerzos de conservación no deberían limitarse sólo a áreas de ecosistemas naturales, sino también a áreas rurales, urbanas y suburbanas (Daily et al. 2003; Rosenzweig 2003).

Los procesos de urbanización en Colombia, y particularmente en la Sabana de Bogotá, han causado cambios drásticos en los ecosistemas. La Sabana de Bogotá durante el Pleistoceno fue un sistema de humedales en su zona plana rodeada por bosques andinos y hasta antes de la llegada de los europeos esta situación no cambió considerablemente (Van der Hammen 2003). Hoy la Sabana está invadida por construcciones y su paisaje actual es un mosaico que refleja las diferentes necesidades humanas por refugios, alimento y áreas para la oferta y producción de diferentes bienes y servicios (Camargo Ponce de León 2007). Adicionalmente a la remoción de los ecosistemas nativos, también se ha reemplazado en la Sabana la flora silvestre por especies exóticas como el pasto kikuyo (Cenchrus clandestinum), los pinos (Pinus spp.) y los eucaliptos (Eucalyptus spp.). Las especies exóticas eventualmente pueden naturalizarse, es decir interactuar con especies de fauna nativa e integrarse al funcionamiento del ecosistema (Cavelier y Santos 
1999), pero es poco lo que sabemos sobre cómo la introducción de especies exóticas a la Sabana afecta a su fauna nativa (Sánchez 2010). La información disponible sugiere que los cambios que han ocurrido en la Sabana han causado una reducción en la diversidad de especies de flora y fauna silvestres, y se conserva un limitado número de especies representativas de la mastofauna andina, que toleran las actividades rurales y urbanas, e incluso pueden aprovechar zonas dominadas por especies exóticas (Mendoza 2012).

Por otra parte, para enfrentar la pérdida de biodiversidad a nivel mundial se ha hecho mayor énfasis en los estudios con especies consideradas como vulnerables o en peligro crítico de extinción, y hay relativamente poco interés por las denominadas especies "comunes", las cuales también pueden ser susceptibles a extinciones locales por cambios ambientales (Roger et al. 2007; Lindenmayer et al. 2011). Adicionalmente, el estudio de estas especies comunes, puede ayudarnos a entender por qué pueden tolerar los cambios que los seres humanos hacemos en el ambiente y entender sus requerimientos ecológicos para que coexistan con nosotros (Cáceres 2000; Roger et al. 2007).

En este trabajo exploramos los comportamientos de forrajeo del tlacuache de orejas blancas o fara Didelphis pernigra, que es el mamífero silvestre más grande que sobrevive en las áreas urbanas y suburbanas de la Sabana y es relativamente común en áreas rurales de los Andes. El fara es un marsupial de la familia Didelphidae, orden Didelphimorphia, que se encuentra en los Andes de Colombia, Venezuela, Ecuador, Perú, y Bolivia (Lemos y Cerqueira 2002). En su dieta incluye invertebrados, frutos y eventualmente pequeños vertebrados como lagartijas, aves y ratones (Pérez-Hernández et al. 1994; Durant 2002). En Colombia, D. pernigra se distribuye en altitudes entre los 2,000 y 3,600 m (Alberico et al. 2000), y ha sido registrada en bosques andinos, páramos, y zonas reforestadas con especies nativas y exóticas (López-Arévalo y Montenegro-Díaz 1993; Sánchez y Alvear 2003; Ramírez-Chaves et al. 2008). Hasta donde sabemos, sólo se ha realizado un estudio en Colombia sobre cómo las modificaciones espaciales hechas por los humanos afectan su forrajeo (Suárez 2012). Los estudios del comportamiento de forrajeo pueden brindar información acerca del uso del hábitat por una población y permiten examinar los factores que afectan las decisiones de forrajeo, ya que dependiendo de las características del sitio, los animales escogen donde forrajear, la cantidad de tiempo invertido en la actividad y la cantidad de alimento a consumir (Brown 2000). Por lo anterior, el comportamiento de forrajeo puede usarse como una herramienta para tomar decisiones en estrategias de conservación de hábitats para una especie, dado que ayudan a determinar la calidad de los mismos (Narváez y Sánchez 2013), y la efectividad de programas de conservación (Lindell 2008; Morris et al. 2009).

Una técnica usada para estudiar el uso de hábitat en animales ha sido la medida de densidades de abandono (DDA), que corresponde a la cantidad de alimento dejada por el animal en un parche alimentario después de forrajear (Brown 1988). El uso de DDA's fue propuesto como parte de un modelo de uso óptimo de parches que plantea que un forrajeador permanece en un parche alimentario mientras los beneficios superan los costos de explotarlo. Los beneficios son indicados por la tasa de cosecha al explotar el parche, es decir, la cantidad de alimento obtenida por unidad de tiempo. Los costos de forrajeo incluyen costos asociados con el riesgo de ser depredado, costos energéticos y costos de oportunidades perdidas. Entonces, según el modelo de Brown la tasa de cosecha de un forrajeador en un parche en el que experimenta ganancias decrecientes 
como resultado del consumo del alimento, se relaciona con la cantidad de alimento en el parche, y por ello la cantidad de alimento dejada por el forrajeador está asociada con la tasa de cosecha de abandono (Brown 1988, 1992; Sánchez 2006). El cambio en la tasa de cosecha indica cuándo los beneficios no compensan los costos de forrajeo y refleja el valor marginal del parche para el forrajeador. Por ello, entre más comida abandone el forrajeador en el parche, mayor será la DDA y menor el valor marginal para él.

Con base en lo anterior, utilizamos el modelo de Brown (1988) para examinar el forrajeo y los movimientos del fara $D$. pernigra en un paisaje altamente modificado en la Sabana de Bogotá. El área de estudio, el campus de la Universidad Militar Nueva Granada (UMNG), ha sido afectada por procesos asociados a actividades rurales y de urbanización, hay amplias áreas sembradas con pasto kikuyo (Cenchrus clandestinum), y hay plantaciones de eucalipto (Eucaliptus sp.) y áreas en regeneración natural cerca del río Bogotá. En particular, estudiamos si los faras se movilizaban y forrajeaban por las zonas de pastizal, que representan ambientes altamente modificados, dominados por una especie exótica como el kikuyo y que son podados con regularidad. Por ello en este trabajo buscamos respuesta a las siguientes preguntas: 1) ¿actúan los pastizales como una barrera para el desplazamiento del fara?, y 2) ¿la distancia al río, donde hay bosques en regeneración y plantaciones de eucaliptos, afecta negativamente el forrajeo del fara? Adicionalmente examinamos el posible efecto de la iluminación de la luna, la temperatura del aire, y la precipitación pluvial sobre la actividad de forrajeo del fara.

Área de estudio. Estudiamos el forrajeo de los faras durante dos meses (Noviembre/ Diciembre 2011) en el campus de la UMNG, en la Cordillera Oriental de Colombia, municipio de Cajicá, departamento de Cundinamarca $\left(4.941722^{\circ},-74.015146^{\circ} ; \sim 2,580\right.$ m; Fig. 1). Con base en los datos de la estación meteorológica en el campus, durante el 2011 la temperatura media anual fue $13.5^{\circ} \mathrm{C}$ y la humedad relativa promedio anual fue de $84.7 \%$, el promedio de precipitación mensual fue $173.4 \mathrm{~mm}$, con un patrón bimodal anual, siendo los meses de mayor precipitación mayo y octubre. El campus es un ambiente altamente intervenido con suelos ricos en arcilla, y se puede dividir en tres zonas: 1) zona con edificaciones y pastizales-jardines, 2) zona dominada por pastizales (principalmente C. clandestinum) con árboles dispersos y áreas de cultivo, y 3) otra zona correspondiente a la ronda hídrica del río Bogotá. La zona del río tiene plantaciones de eucaliptos de 10-15 m de altura y áreas de regeneración natural dominadas por romerillos (Baccharis sp.) y sauces (Salix humboldtiana), y en el estrato herbáceo es abundante el kikuyo y adicionalmente hay árboles de aliso (Alnus jorullensis), cedro(Cedrela odorata), y enredaderas como la mora (Rubus sp.) y ojo de poeta (Thunbergia alata).

El estudio del forrajeo lo realizamos en dos trayectos aproximadamente paralelos en la zona dominada por pastizales (Fig. 1), separados cada uno por $\sim 600 \mathrm{~m}$. El trayecto 1 es una zona inundable, regularmente podada y presenta una hilera de eucaliptos a manera de cerca viva, a $\sim 50 \mathrm{~m}$ de un cultivo de flores en el lote vecino y finaliza en un vallado de $\sim 1 \mathrm{~m}$ de profundidad e inicio de la zona de ronda del río Bogotá. En el trayecto 2, el herbazal es denso y a 10 metros de distancia de los cultivos experimentales del campus (principalmente de clavel, fresas, lulo, y tomate) y se ubica a $\sim 10 \mathrm{~m}$ de distancia de un edificio. El trayecto 2 no presenta árboles sino hasta en las cercanías al río Bogotá, y 
había postes de luz eléctrica, los cuales fueron usados para fijar los comederos.

Además aproximadamente a 3 m de distancia, en el límite con la finca vecina, hay una cerca metálica y electrificada.

Comportamientos de forrajeo. Para examinar el comportamiento de forrajeo del fara instalamos seis estaciones de forrajeo en cada uno de los dos trayectos, con una distancia de entre 30 y 40 m entre estaciones consecutivas. Dado que el fara parece consumir frutas con regularidad en la Sabana de Bogotá (F. Sánchez obs. pers.), usamos como estaciones de forrajeo contenedores cilíndricos plásticos de $6 \mathrm{~cm}$ de diámetro y $14.5 \mathrm{~cm}$ de altura, con $150 \mathrm{ml}$ de una mezcla de agua con azúcar (relación 1:3) y 60 esferas de vidrio (1.5 cm de diámetro) como sustrato sólido, para dificultar la obtención de alimento al profundizar en el comedero y así generar ganancias decrecientes (Sánchez 2006). Este diseño ya había sido usado con éxito en otra área de la Sabana de Bogotá (Suárez 2012). Las estaciones fueron protegidas de la lluvia con un techo de plástico y reforzadas en su base con cinta pegante gruesa. En cada estación utilizamos tubos de PVC de 2 pulgadas de diámetro y $15 \mathrm{~cm}$ de alto como soporte para los comederos. Colocamos los comederos en árboles o postes de luz eléctrica a alturas entre 0 y $80 \mathrm{~cm}$ de altura, dependiendo de las condiciones del lugar. Durante 15 días habituamos a los faras a que visitaran las estaciones y consumieran el alimento líquido. Para atraerlos usamos inicialmente banano y posteriormente sólo esencia de banano. Seguido al período de habituación, registramos las densidades de abandono de los faras durante 30 días. Así, el esfuerzo de muestreo total fue de 12 estaciones $\times 30$ días.

Durante el período de registro de DDA's usamos sólo esencia de banano como atrayente. El muestreo diario incluyó la preparación del alimento líquido, el pesaje de los comederos con y sin alimento líquido y finalmente, la distribución de los comederos en las estaciones de los dos trayectos antes del atardecer, entre 17:30 y 18:00 $\mathrm{h}$ aproximadamente. Medimos las DDA's en la mañanas entre 07:00 h y 08:00 h, y durante este proceso retiramos los contenedores de las estaciones, rectificamos el número de esferas de vidrio en cada contenedor, pesamos el comedero con alimento y restamos el peso del comedero con las esferas para obtener la cantidad de alimento en el comedero, es decir, la DDA. Dado que la evaporación podría reducir las DDA's, dejamos un comedero con una malla en la parte superior, para que no pudiera ser usado por los faras en cada trayecto y posterior al periodo de habituación encontramos que el efecto era despreciable ( $<0.5 \mathrm{~g} /$ noche). Colocamos cinta doble faz en los comederos para que los pelos de los faras quedaran pegados y sirvieran como prueba de la visita. De los faras capturados (ver adelante) obtuvimos pelos para comparar con los encontrados en los comederos. La precipitación, temperatura máxima y mínima, y humedad relativa de cada noche de muestreo los obtuvimos de la estación meteorológica del campus y la iluminación de la lunar con el programa de libre distribución Moonphase 3.3 (Tingstrom 2009).

Registro Directo. De manera simultánea a la evaluación del forrajeo, capturamos faras con el fin de verificar su presencia en el campus. Para la captura de faras usamos trampas Tomahawk y Duke, $30 \times 25 \times 70 \mathrm{~cm}$, dispuestas de manera aleatoria en la plantación de eucalipto cercana al río para no interferir con los experimentos de forrajeo (Fig. 1). Se emplearon 4 trampas durante cuatro noches, dispuestas entre las 16:00-18:00 h y 
revisadas entre las 06:00-08:00 h del día siguiente. El cebo utilizado en las trampas fue banano y mandarina. Usamos un esfuerzo total de 64 trampas/noche. Los individuos capturados se marcaron en la oreja derecha con números consecutivos empleando un tatuador permanente para conejos, se registraron las medidas estándares para mamíferos, sexo y peso, posteriormente los dejamos en libertad en el sitio de captura (Jones et al. 1996). El éxito de captura lo calculamos como el número de individuos capturados dividido por el esfuerzo de captura multiplicado por 100.

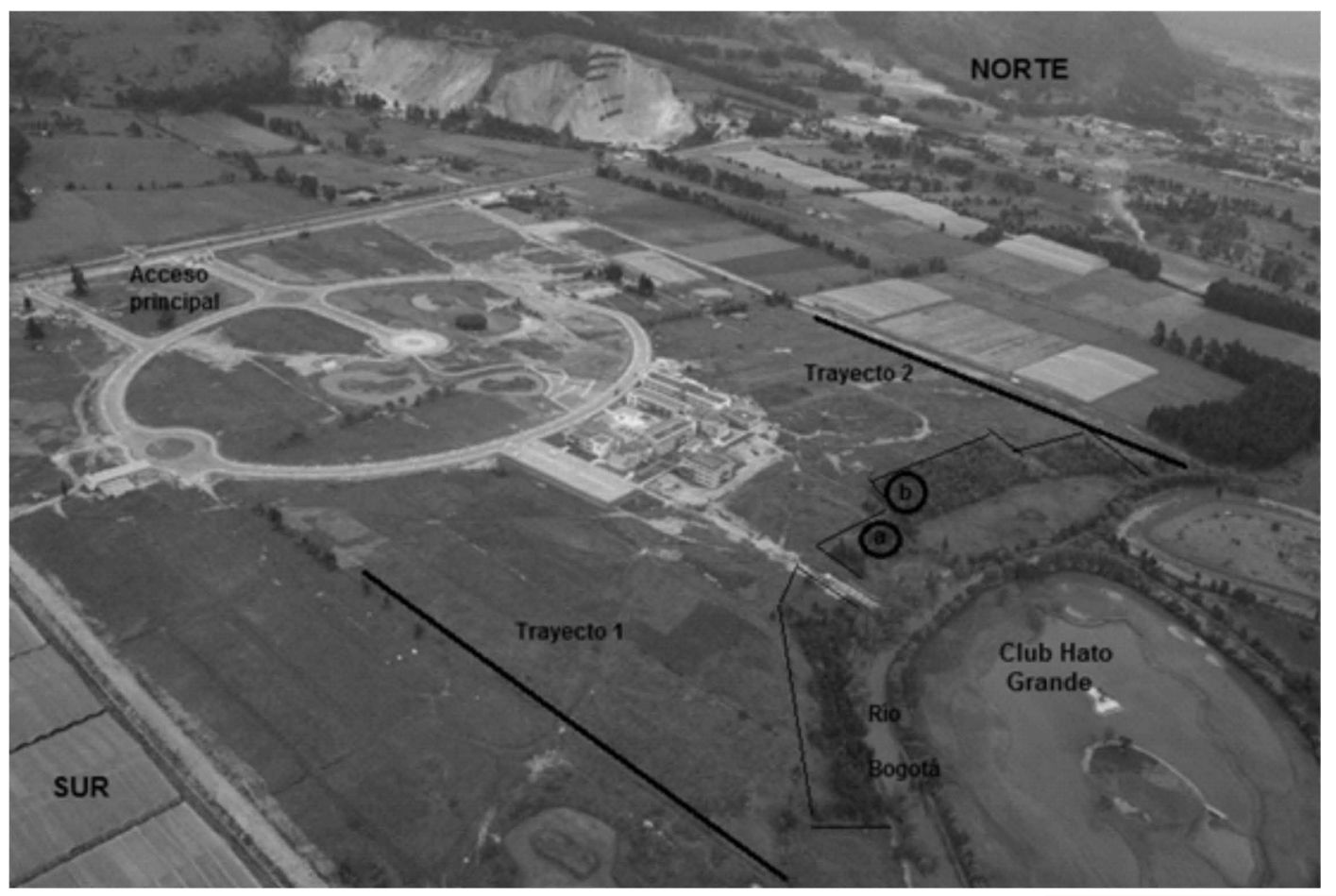

Figura 1.Campus de la UMNG, Cajicá, Colombia. Se muestran los trayectos en los que se distribuyeron las estaciones de forrajeo (trayectos 1 y 2 ) y la zona de plantación de eucalipto donde se realizaron los trampeos (círculos a y b). El costado oriental del campus está rodeado por el río Bogotá. Cortesía Departamento de Planeación UMNG, fotografía aérea, 2011.

Análisis de datos. Para estudiar el forrajeo de los faras en los dos trayectos del campus utilizamos un modelo lineal generalizado con función gamma, con las densidades de abandono como variable dependiente y las variables independientes fueron los trayectos, las estaciones, y la iluminación de la luna. Escogimos este modelo estadístico dado que se violaban los supuestos de normalidad y homoscedasticidad requeridos por modelos paramétricos (Zar 1999). En el modelo final incluimos únicamente los factores que afectaron significativamente al forrajeo de acuerdo con el método de Wilson y Hardy (2002). Adicionalmente usamos análisis de correlación no paramétricos de Spearman (Zar 1999) para examinar los posibles efectos de la iluminación de la luna, precipitación, la humedad relativa y las temperaturas máxima y mínima del aire sobre el forrajeo de los faras. Evaluamos las hipótesis estadísticas con un nivel de significancia $\alpha=0.05$.

\section{Resultados}

Comportamiento de forrajeo. El modelo lineal generalizado indicó que las variables trayecto $\left(\mathrm{c}^{2}\right.$ Wald $=6.99 ;$ g. I. $\left.=1 ; P=0.008\right)$, trayecto $\times$ estación $\left(\mathrm{c}^{2}\right.$ Wald $=20.56$; g. I. = $10 ; P=0.024)$ y día $\left(c^{2}\right.$ Wald $=84.31 ;$ g. I. $\left.=24 ; P<0.001\right)$ afectaron el forrajeo del fara. Las DDA's en trayectos y estaciones mostraron gran variabilidad, y entre las estaciones dentro de cada trayecto no se evidenciaron diferencias significativas (Fig. 2). 
Sin embargo, dos estaciones en el trayecto 2 presentaron DDA's más altas que todas las estaciones del trayecto 1 , es decir, que fueron poco explotadas por los faras. Estas dos estaciones estaban localizadas en los extremos del trayecto, es decir la más cercana al río, estación 1 y la más lejana a él, estación 6.

También se encontró una alta variabilidad en las DDA's a nivel temporal (Fig. 3), pero reconocimos que en algunos días hubo bajas DDA's como el día 17 del muestreo, y otros días los faras tuvieron altas DDA's, como el día 5. Para analizar el efecto temporal sobre el forrajeo de los faras, empleamos correlaciones no paramétricas entre la iluminación de la luna y las DDA's, y algunas variables climáticas y las DDA's. Encontramos que la iluminación de la luna tuvo una correlación positiva y significativa (Spearman Rho = $0.155, P=0.003)$ con las DDA's. Dado que las DDA's están inversamente relacionadas con el valor marginal de los parches alimentarios, este resultado indica que la relación beneficios/costos del forrajeo de los faras mejora cuando las noches son más oscuras. Las variables humedad relativa (Spearman Rho $=-0.053, P=0.316$ ), y temperatura máxima (Spearman Rho $=-0,042, P=0.382$ ) y mínima del aire (Spearman Rho $=-0.023, P=$ 0.253) no tuvieron efecto sobre el forrajeo del fara. Adicionalmente se evidenció una correlación positiva, pero no significativa, entre la precipitación y las DDA's (Spearman Rho $=0.091, P=0.086$ ).

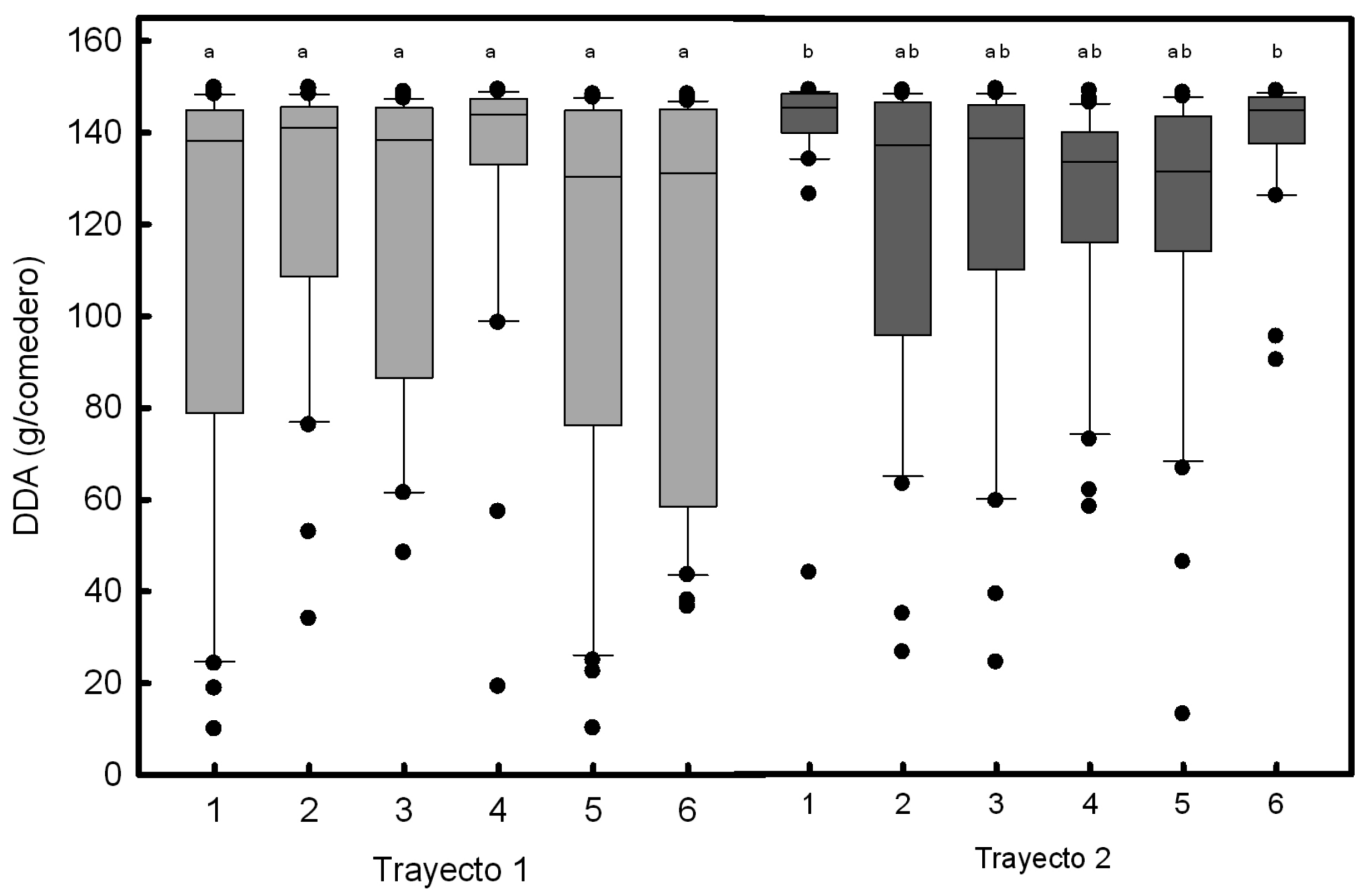

Figura 2. Forrajeo de los faras (Didelphis pernigra) en parches alimentarios, medido en densidades de abandono (DDA), a lo largo de dos trayectos con seis estaciones en áreas de pastizales y árboles; al aumentar el número del parche alimentario (eje de las abscisas) disminuye la distancia con respecto a una zona arbolada en la ronda del río Bogotá, en el campus Cajicá de la UMNG. No hubo diferencias entre las estaciones del mismo trayecto, pero las estaciones 1 y 6 del trayecto 2, mostraron DDA's significativamente superiores a todas las estaciones del trayecto 1. La línea dentro de la caja es la mediana, sus extremos son los percentiles 25 y 75, la barra de error representa los percentiles 10 y 90 , y los puntos son valores atípicos. Las letras diferentes indican diferencias significativas ( $c^{2}$ Wald, $\left.P<0.05\right)$.

Registro Directo. Capturamos dos faras macho en el campus, para un éxito de captura de $3.13 \%$. Los faras fueron capturados en el mismo lugar (círculos a y b de Fig. 1), en 
un área con abundante mora (Rubus sp.) y kikuyo, rodeado de árboles de eucalipto. Las medidas somáticas convencionales (en $\mathrm{mm}$ ) de los faras fueron: individuo con el tatuaje 1, longitud total (LT) 790, longitud de la cola (LC) 351, longitud de la pata (LP) 62, longitud de la oreja (LO) 37, peso 1,700 g, e individuo con el tatuaje 2, LT 850, LC 306, LP 62, LO 47, peso 1,600 g. Adicionalmente, durante el tiempo del estudio, 4 cadáveres de faras fueron encontrados a lo largo del trayecto 2, entre las estaciones 3 y 6 .

Discusión

Esperábamos encontrar que el forrajeo y movilización de los faras en el campus fuera afectado por las zonas de pastizal y la distancia a la zona arbolada cerca al río Bogotá. Sin embargo, nuestros resultados sugieren que ni el pastizal es una barrera para el forrajeo del fara, ni el uso de parches alimentarios se relaciona con la distancia al río. Esto refuerza la idea de que los miembros del género Didelphis toleran ambientes altamente modificados y los resultados muestran que $D$. pernigra puede movilizarse y/o forrajear por áreas desprovistas de vegetación nativa y poca vegetación arbustiva o arbórea. Otros estudios con diferentes especies de la familia Didelphidae indican que estos marsupiales hacen uso de zonas dominadas por árboles exóticos como el eucalipto (Aguirre et al. 1998; Mendoza 2012), pero hasta donde sabemos, esta es la primera vez que se reconoce el forrajeo del fara en ambientes dominados por especies exóticas no arbóreas, como el kikuyo. El fara habita en ambientes naturales abiertos como áreas de páramo en Colombia y Venezuela (López-Arévalo y Montenegro-Díaz 1993; Durant 2002) y probablemente su capacidad para explotar este ecosistema y sus recursos le ha permitido aprovechar las zonas de pastizal presentes en el campus.

La técnica de las DDA's puede usarse para reconocer cómo los forrajeadores hacen uso del espacio y si distinguen diferencias a nivel de hábitat o microhábitat (Brown et al. 1994; Narváez y Sánchez 2013). La ausencia de diferencias en el forrajeo al interior de los trayectos sugiere que, desde el punto de vista de los faras, la zona dominada por pastizales es un único hábitat, y sólo hay diferencias cuando se examinan estaciones particulares, i.e., a nivel de microhábitat. Esto concuerda con lo encontrado en algunas especies de marsupiales australianos de tamaño mediano, en las que el uso de hábitat depende de la escala espacial, y también de la hora del día (Finlayson et al. 2008). Según Finlayson et al. (2008), algunos marsupiales son más selectivos en el uso del espacio al momento de escoger donde refugiarse durante el día y no demuestran preferencias por algún tipo de cobertura durante el período de forrajeo en la noche. Para examinar si algo similar ocurre en el campus de la UMNG sería necesario extender los esfuerzos para capturar y registrar el forrajeo de los faras a otras coberturas además del pastizal, pero parece que los faras seleccionan sitios de descanso, ya que hemos observado animales en árboles de la ronda del río, y nunca descansando en la zona de pastizales o las zonas construidas.

El modelo de uso óptimo de parches de Brown (1988) asume que la explotación de un parche alimentario depende de la relación entre costos y beneficios percibida por el forrajeador. Debido a que en todas las estaciones se usaba el mismo tipo de comedero, no había diferencias en los costos energéticos; de igual manera, el tiempo de muestreo fue relativamente corto dentro del ciclo reproductivo de los faras (TyndaleBiscoe y Mackenzie 1976), por lo tanto, los costos asociados a oportunidades perdidas 
probablemente tendrían poco impacto a lo largo de nuestro trabajo. Así, los costos de forrajeo que podrían afectar con mayor intensidad el forrajeo de los faras estarían asociados al costo percibido de ser depredados (Brown y Kotler 2007). En este contexto, nuestros resultados sugieren que para los faras no hubo un incremento en el riesgo percibido de depredación al adentrarse en el pastizal, y probablemente no hay depredadores que intimiden lo suficiente al fara para afectar sus decisiones de forrajeo. Se ha encontrado que los principales agentes de mortalidad para Didelphis en áreas sub-urbanas son los automóviles, además de depredadores naturales o exóticos como los perros domésticos (Cáceres 2000). Así, la ausencia o baja frecuencia de automóviles y perros pueden explicar nuestros resultados. En el campus hemos observado depredadores nocturnos como búhos (Pseudoscops clamator) y lechuzas (Tyto alba), pero sus dietas incluyen invertebrados y pequeños vertebrados que generalmente no superan los $500 \mathrm{~g}$ (Huston y Nelson 1994; Delgado-V. et al. 2005). Esto sugiere que es improbable que estas rapaces puedan representar un riesgo para faras adultos que pesan más de 1000 g, y así causen que eviten zonas abiertas, como pastizales, pero sería necesario examinar si pueden afectar a animales juveniles.

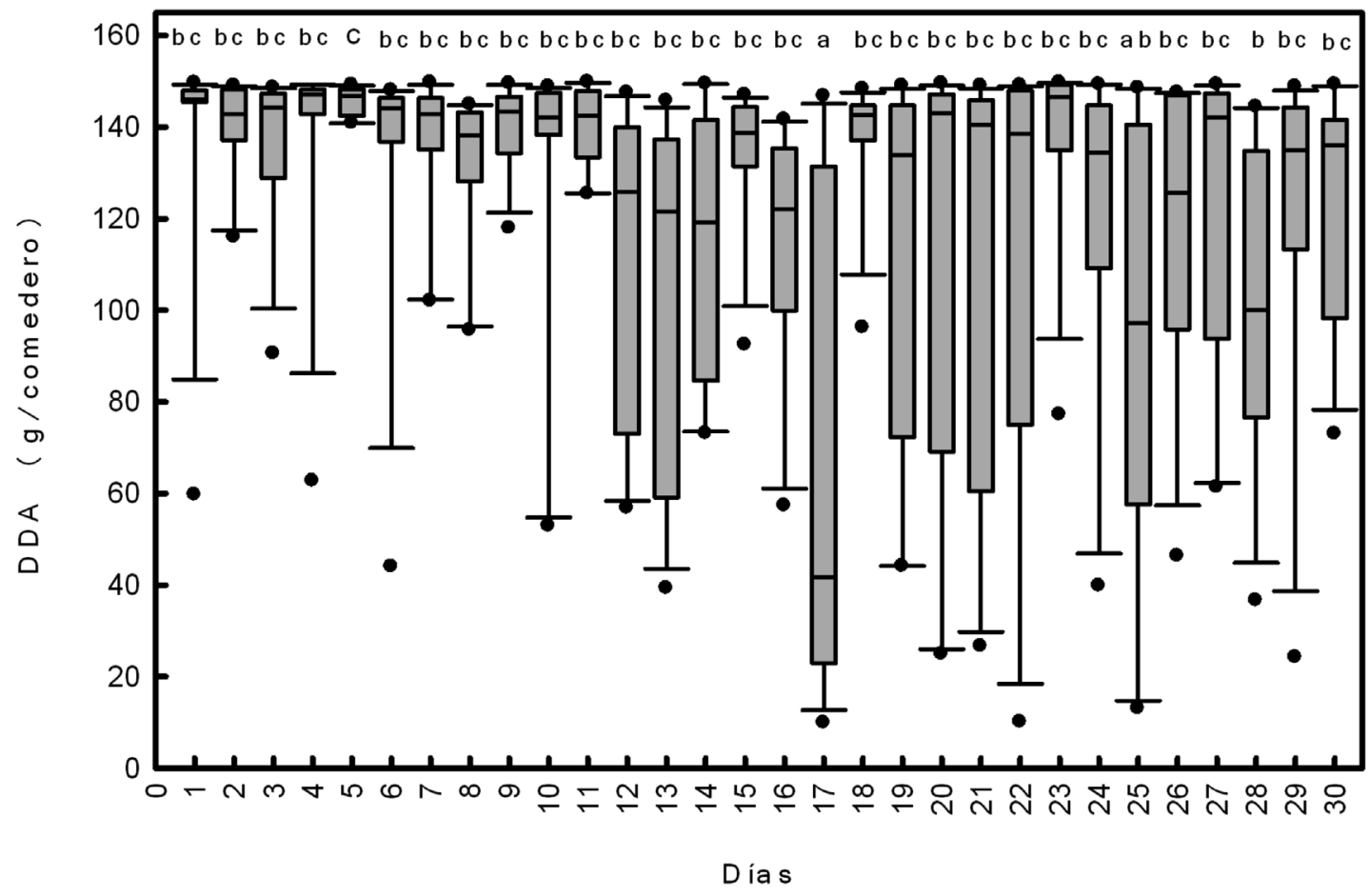

Figura 3. Variación del forrajeo de los faras Didelphis pernigra, medido en densidades de abandono (DDAs), con respecto al tiempo de experimentación. No hubo diferencias significativas entre las DDAs, excepto el día 5 que presentó un valor más bajo que los días 18 y 28, y el día 17 tuvo un valor más alto que el resto de días. La línea dentro de la caja es la mediana, sus extremos son los percentiles 25 y 75, la barra de error representa los percentiles 10 y 90 , y los puntos son valores atípicos. Las letras diferentes indican diferencias significativas ( $c^{2}$ Wald, $\left.P<0,05\right)$.

Como mencionamos, detectamos un efecto de microhábitat indicado por la interacción significativa entre trayecto y estación de forrajeo, debido específicamente a la baja explotación de dos estaciones en el trayecto 2 respecto a las estaciones del trayecto 1. La mayoría de las estaciones en el trayecto 2 se fijaron a postes de luz, pero sólo uno iluminaba durante toda la noche, y ésta estación fue menos explotada que las del 
trayecto 1. Así, este resultado posiblemente sea consecuencia de la luz artificial, que en mamíferos silvestres puede reducir sus movimientos y/o actividad (ver Rich y Longcore 2002). Adicionalmente, la correlación positiva entre las DDA's y la iluminación de la luna, indica que entre más oscura la noche mayor el valor marginal del alimento. En otras especies de mamíferos también se ha evidenciado una preferencia a forrajear durante las noches con bajos niveles de iluminación lunar (Orrock et al. 2004). Estos resultados se han asociado generalmente con el riesgo de depredación percibido (Kotler et al. 1994), y se asume que los animales quedan más expuestos en noches más iluminadas. Sin embargo, nuestros resultados contrastan con lo encontrado por Norris et al. (2010), donde D. marsupialis, en un área fragmentada de Brasil mostró una mayor actividad al aumentar los niveles de iluminación lunar posiblemente porque mejoraba su visibilidad, de acuerdo con los autores.

No es claro si el menor forrajeo al aumentar la iluminación del sitio es por un mayor riesgo de depredación percibido por los faras dentro del campus o si este comportamiento sea innato y producto de presiones selectivas a lo largo de varias generaciones. Sin embargo, los resultados de este estudio sugieren que por ejemplo, las áreas alrededor de las edificaciones con alta iluminación serían menos usadas por los faras. Así, el aumentar la cantidad de construcciones, como se tiene planeado en el campus, posiblemente tenga un impacto negativo en el comportamiento del fara al reducir el valor de las zonas iluminadas. Por otro lado, la segunda estación menos explotada del trayecto 2 se encontraba cercana al río. Observamos durante el tiempo de habituación y hasta el tercer día de experimentación, que el parche era explotado por los faras, y luego de ese día el uso del parche decreció considerablemente. La reducción en el uso de esta estación coincidió con el aumento en el uso de máquinas ruidosas como motobombas y motosierras en el lote vecino. Así, nuestros resultados parecen concordar con los encontrados en un bosque natural en Estados Unidos, donde se demostró que las ardillas de cola roja responden con cambios en el uso del espacio por la intrusión humana, disminuyendo su forrajeo y alejándose de los territorios perturbados (Gutzwiller y Riffell 2008).

También encontramos una considerable variabilidad temporal en el uso de los comederos. Como mencionamos anteriormente, parte de dicha variación es explicada por el efecto de la iluminación de la luna, pero probablemente otros factores son responsables de la alta variación temporal encontrada. Factores como la humedad relativa, y las temperaturas máxima y mínima no tuvieron efecto en el forrajeo, y la precipitación no alcanzó a ser significativa, aunque hubo una tendencia de correlación positiva con respecto a las DDA's. En D. marsupialis y D. albiventris se ha encontrado que la precipitación afecta las actividades propias del desarrollo de los individuos y aumenta la competencia y el territorialismo (Durant 2002; Passamani y Ribeiro 2009). Por esto serían necesarios muestreos más prolongados para evaluar el posible efecto de la lluvia.

A manera de conclusión, los faras, al igual que otros miembros del género Didelphis, son especies capaces de explotar múltiples tipos de hábitat, incluyendo aquellos altamente modificados por el ser humano. Los Didelphis potencialmente son controladores de poblaciones de invertebrados, y su consumo de frutas sugiere que pueden actuar como dispersores de semillas (Cáceres 2002; Passamani y Ribeiro 2009). Además, varias 
especies de Didelphis pueden moverse cientos de metros e incluso kilómetros en una sola noche (Sunquist et al. 1987; Loretto y Vieira 2005) y es posible que ese sea también el caso del fara. Así, la conservación del fara en el campus podría ser importante por las mismas razones que se han reconocido para otras especies comunes (ver referencias en Lindenmayer et al. 2011): por su participación en procesos ecológicos, porque son las especies más probables a adaptarse a los cambios generados por los humanos, pueden ser indicadores de los mismos cambios y puede ser práctico su seguimiento y estudio, como lo hemos demostrado en este trabajo. Para el caso particular del campus de la UMNG, y buena parte de la Sabana de Bogotá, la tolerancia del fara a espacios altamente modificados $y$, posiblemente su alta movilidad, sugieren que este marsupial podría ayudar a mantener funciones ecosistémicas y podría favorecer procesos de restauración ecológica en lugares deteriorados donde otras especies silvestres rara vez se aventurarían.

\section{Agradecimientos}

Agradecemos a la UMNG por permitir los muestreos dentro del campus y por su apoyo. A los estudiantes de Biología Aplicada por su colaboración y ánimo en la fase de muestreos, especialmente a L. Mendoza, R. Rodríguez y H. Sánchez. El laboratorio de Ecología y Conservación Ambiental de la UDCA facilitó las trampas usadas para la captura de faras. Finalmente, agradecemos la revisión juiciosa de dos evaluadores anónimos que ayudaron a mejorar la calidad del manuscrito.

\section{Literatura citada}

Aguirre, L. F., J. A. Balderrama, C. F. Pinto, E. I. Maradiegue, y R. Vargas. 1998. Influencia de dos especies forestales exóticas sobre fauna terrestre de bosques nativos de kewiña en el Parque Nacional Tunari. Conservación de Bosques Nativos 5:14021406.

Alberico, M., A. Cadena, J. Hernández-Camacho, y Y. Muñoz-Saba. 2000. Mamíferos (Synapsida: Theria) de Colombia. Biota Colombiana 1:43-75.

Armenteras, D., F. Gast, y H. Villareal. 2003. Andean forest fragmentation and the representativeness of protected natural areas in the eastern Andes, Colombia. Biological Conservation 113:245-256.

Brown, J. S. 1988. Patch use as an indicator of habitat preference, predation risk, and competition. Behavior, Ecology and Sociobiology 22:37-47.

Brown, J. S. 1992. Patch use under predation risk: I. Models and predictions. Annales Zoologici Fennici 29:301-309.

Brown, J. S., B. P. Kotler, y W. A. Mitchell. 1994. Foraging theory, patch use, and the structure of a Negev Desert granivore community. Ecology 75:2286-2300.

Brown, J. S. 2000. Foraging ecology of animals in response to heterogeneous environments. Pp. 181-215 en The Ecological Consequences of Environmental Heterogeneity (Hutchings J., y A. Stewart, eds.). Blackwell Scientific. Oxford, Reino Unido.

Brown, J. S., y B. P. Kotler. 2007. Foraging and the ecology of fear. Pp. 437-480 in Foraging (Stephens, D. W., J. S. Brown, y R. Ydenberg, eds.). University of Chicago Press. Chicago, EE.UU. 
Cáceres, N. C. 2000. Population ecology and reproduction of the white-eared opossum Didelphis albiventris (Mammalia: Marsupialia) in an urban environment of Brazil. Ciência e Cultura Journal of the Brazilian Association for the Advancement of Science 52:171-174.

CÁceres, N. C. 2002. Food habits and seed dispersal by the white-eared opossum Didelphis albiventris in Southern Brazil. Studies on Neotropical Fauna and Environment 37:97-104.

Camargo Ponce de León, G. 2007. Estado y perspectivas de los ecosistemas urbanos de Bogotá. Prioridades 2008-2011. Foro Nacional Ambiental 16:1-8.

Cavelier, J., y C. Santos. 1999. Efectos de plantaciones abandonadas de especies exóticas y nativas sobre la regeneración natural de un bosque montano en Colombia. Revista de Biología Tropical 47:775-784.

Daily, G. C., G. Ceballos, J. Pacheco, G. Suzan, y A. Sanchez-Azofeifa. 2003. Countryside biogeography of neotropical mammals: Conservation opportunities in agricultural landscapes of Costa Rica. Conservation Biology 17:1814-1826.

Delgado-V., C. A., P. C. Pulgarín-R., y D. Calderón-F. 2005. Análisis de egagrópilas del búho rayado (Asio clamator) en la ciudad de Medellín. Ornitología Colombiana 3:100-103.

Durant, P. 2002. Notes on white-eared opossum Didelphis albiventris Lund, 1840 from Mérida Andes, Venezuela. Revista de Ecología Latino Americana 9:1-7.

Etter, A., y W. van Wyngaarden. 2000. Patterns of landscape transformation in Colombia, with emphasis in the Andean region. Ambio 29:432-439.

Etter, A., y L. A. Villa. 2000. Andean forrests and farming systems in part of the Eastern Cordillera (Colombia). Mountain Research and Development 20:236-245.

Finlayson, G. R., E. M. Vieira, D. Priddel, R. Wheeler, J. Bentley, y C. R. Dickman. 2008. Multi-scale patterns of habitat use by re-introduced mammals: a case study using medium-sized marsupials. Biological Conservation 141:320-331.

Gutzwiller, K. J., y S. K. Riffell. 2008. Does human repeated intrusion alter use of wildland sites by red squirrels? Multiyear experimental evidence. Journal of Mammalogy 89:374-380.

Huston, R. M., y T. A. Nelson. 1994. Barn owl (Tyto alba) food habits in west-central Arkansas. Proceedings Arkansas Academy of Science 48:73-74.

Jones, C., W. J. Mcshea, M. J. Conroy, y T. H. Kunz. 1996. Capturing Mammals. Pp 115-155 en Measuring and monitoring biological diversity standard methods for mammals. (Wilson, D. E., F. R. Cole, J. Nichols, R. Rudran, y M. S. Foster, eds.). Biological diversity handbook series. Smithsonian Institution Press. London, Reino Unido.

Kotler, B. P., J. S. Brown, y W. A. Mitchell. 1994. The role of predation in shaping the behavior, morphology and community organization of desert rodents. Australian Journal of Zoology 42:449-466.

Lemos, B., y R. Cerqueira. 2002. Morphological differentiation in the white-eared opossum group (Didelphidae: Didelphis). Journal of Mammalogy 83:354-369.

LiNDELL, C. A. 2008. The value of animal behavior in evaluations of restoration success. Restoration Ecology 16:197-203. 
Lindenmayer, D. B., J. T. Wood, L. McBurney, C. MacGregor, K. Youngentob, y S. C. BANKs. 2011. How to make a common species rare: a case against conservation complacency. Biological Conservation 144:1663-1672.

López-Arévalo, H. F., y O. L. Montenegro-Díaz. 1993. Mamíferos no voladores de Carpanta. Pp. 165-187 en Carpanta: Selva nublada y páramo (Andrade, G. I., ed.). Fundación Natura. Bogotá, Colombia.

Loretto, D., y M. V. Vieira. 2005. The effects of reproductive and climatic seasons on movements in the black-eared opossum (Didelphis aurita Wied-Neuwied, 1826). Journal of Mammalogy 86:287-293.

Mendoza, L. X. 2012. Composición, riqueza y abundancia de mamíferos de la Hacienda Las Mercedes, Bogotá, Colombia. Tesis de pregrado, Biología, Universidad Militar Nueva Granada. Cajicá, Colombia.

Morris, D. W., B. P. Kotler, J. S. Brown, V. Sundararaj, y S. B. Ale. 2009. Behavioral indicators for conserving mammal diversity. Annals of the New York Academy of Sciences 1162:334-356.

NarváEz, G., y F. Sánchez. 2013. Using foraging behavior to detect edge effects: a case study with Colombian Andean mammals. Boletín Científico del Centro de Museos - Universidad de Caldas 17:110-120.

Norris, D., F. Michalski, y C. A. Peres. 2010. Habitat patch size modulates terrestrial mammal activity patterns in Amazonian forest fragments. Journal of Mammalogy 91:551-560.

Orrock, J. L., B. J. Danielson, y R. J. Brinkerhoff. 2004. Rodent foraging is affected by indirect, but no by direct, cues of predation risk. Behavioral Ecology 15:433-437.

Passamani, M., y D. Ribeiro. 2009. Small mammals in a fragment and adjacent matrix in southeastern Brazil. Brazilian Journal of Biology 69:305-309.

Pérez-Hernández, R., P. Soriano, y D. Lew. 1994. Marsupiales de Venezuela. Cuadernos Lagoven. Caracas, Venezuela.

Puluın, A. S. 2002. Conservation Biology. Cambridge University Press. Cambridge, Reino Unido.

Ramírez-Chaves, H. E., W. Pérez, y J. Ramírez-Mosquera. 2008. Mamíferos presentes en el municipio de Popayán, Cauca-Colombia. Boletín Científico Museo de Historia Natural Universidad de Caldas 12:65-89.

Rich, C., y T. Longcore. 2002. Ecological consequences of artificial night lighting. Program and Abstracts. The Urban Wildlife Group and UCLA Institute of the Environment. Los Angeles, EE.UU.

Roger, E., S. W. Laffan, y D. Ramp. 2007. Habitat selection by the common wombat (Vombatus ursinus) in disturbed environments: implications for the conservation of a 'Common' species. Biological Conservation 137:437-449.

Rosenzweig, M. L. 2003. Win-win ecology: how Earth's species can survive in the midst of human enterprise. Oxford University Press. Oxford, Reino Unido.

SÁnchez, F. 2006. Harvest rates and patch use strategy of Egyptian fruit bats in artificial food patches. Journal of Mammalogy 87:1140-1144.

SÁnchez, F. 2010. Mamíferos pequeños. Pp. 173-188 in Proyecto corredor borde norte de Bogotá. Fase I (Ardila, G., ed.). Instituto de Estudios Urbanos - Universidad Nacional de Colombia, Universidad de Ciencias Aplicadas y Ambientales, Academia 
Colombiana de Ciencias Exactas Físicas y Naturales, Instituto Geográfico Agustín Codazzi y Corporación Autónoma Regional de Cundinamarca - CAR. Disponible en http://www.car.gov.co/?idcategoria=18115.

Sánchez, F., y M. Alvear. 2003. Comentarios sobre el uso de hábitat, dieta y conocimiento popular de los mamíferos en un bosque andino de Caldas, Colombia. Boletín Científico del Museo de Historia Natural - Universidad de Caldas 7:121-144.

Suárez, G. 2012. Ecología de forrajeo del fara Didelphis pernigra (Didelphidae, Didelphimorphia) en un área suburbana del norte de Bogotá, Colombia. Tesis de pregrado, Profesional en Ciencias Ambientales, Universidad de Ciencias Aplicadas y Ambientales. Bogotá, Colombia.

Sunquist, M. E., S. N. Austad, y F. Sunquist. 1987. Movement patterns and home range in the common opossum (Didelphis marsupialis). Journal of Mammalogy 68:173176.

Tingstrom, H. 2009. Moonphase 3.3. Programa distribuido por Tingam. Disponible en www.tingam.com.

Tyndale-Bıscoe, C. H., y R. B. Mackenzie. 1976. Reproduction in Didelphis marsupialis and D. albiventris in Colombia. Journal of Mammalogy 57:249-265.

Van der Hammen, T. 2003. Los humedales de la Sabana: origen, evolución, degradación y restauración. Pp. 19-51 in Los humedales de Bogotá y la Sabana (Güarnizo A., y B. Calvachi, eds.). Acueducto de Bogotá - Conservación Internacional, Bogotá, Colombia.

Wilson, K., y I. C. W. Hardy. 2002. Statistical analysis of sex ratios: an introduction. Pp. 48-92 en Sex ratios: concepts and research methods (Hardy, I. C. W., ed.). Cambridge University Press. Cambridge, Reino Unido.

ZaR, J. H. 1999. Biostatistical analysis. 4th ed. Prentice Hall, Upper Saddle River. New Jersey, EE.UU.

Sometido: 14 de noviembre de 2013

Revisado: 31 de enero de 2014

Aceptado: 19 de febrero de 2014

Editor asociado: Consuelo Lorenzo

Diseño gráfico editorial: Gerardo Hernández 